\title{
Lipocalin-2 is an inflammatory biomarker associated with metabolic abnormalities in Egyptian obese children
}

\author{
Moushira Erfan Zaki ${ }^{1}$, Hala El-Bassyouni ${ }^{2}$, Eman Youness ${ }^{3}$, Nadi Mohamed ${ }^{3}$ \\ ${ }^{1}$ Medical Research Division, Biological anthropology, ${ }^{2}$ Clinical Genetics Department, Human Genetics and Genome Research Division, National Research \\ Centre, Cairo, Egypt. ${ }^{3}$ Medical Biochemistry Department, Medical Research Division, National Research Centre, Cairo, Egypt.
}

\begin{tabular}{l} 
ARTICLE INFO \\
\hline Article history: \\
Received on: 06/07/2014 \\
Revised on: 19/10/2014 \\
Accepted on: $22 / 01 / 2015$ \\
Available online: $27 / 05 / 2015$ \\
\hline Key words: \\
Lipocalin-2, metabolic \\
parameters, obesity, Egyptian \\
children.
\end{tabular}

\begin{abstract}
Background: The aim of the study was to explore the relationships of lipocalin-2 with metabolic risk factors in obese prepubertal children.

Methods: The aim of the study is to examine relationship between serum lipocalin-2 concentrations and metabolic risk factors in 80 obese children and compared with 80 age and sex matched non-obese children controls. Anthropometric and biochemical parameters were measured by standard procedures. Insulin resistance was determined by Homeostasis Model Assessment of Insulin Resistance (HOMA-IR).

Results: In the present cross -section study serum lipocalin-2, leptin, insulin, HOMA-IR blood pressure, triglycerides and low-density lipoprotein (LDL-c) and cholesterol were significantly higher in obese children than non-obese, whereas high-density lipoprotein cholesterol (HDL-c) was significantly lower. After adjustment of lipocalin-2 levels for BMI, age and gender, the correlation between serum lipocalin-2 with weight, waist circumference, body fat $\%$ and metabolic variables such as fasting insulin; glucose and HOMA-IR triglycerides, cholesterol, LDL-C and HDL-C remained significant. The stepwise multiple regression analysis showed that lipocalin-2 levels were independently associated with LDL-c, total cholesterol, triglycerides and HOMA-IR.

Conclusions: lipocalin-2 was significantly elevated in prepubertal obese children and was independently associated with adiposity parameters and its related metabolic complications. The present findings suggest that the measurement of this biomarker may be useful for evaluating metabolic syndrome in Egyptian obese children.
\end{abstract}

\section{INTRODUCTION}

Obesity is a major risk factor for insulin resistance. It is well known that adipocytes could secrete a variety of biologically adipokines, which are thought to contribute to the development of insulin resistance. Lipocalin-2 is secreted by adipocytes and immune cells such as neutrophils and macrophages and was initially described as playing a role in innate immunity, perhaps by sequestrating iron. Obesity is the most common risk factor for insulin resistance, type 2 diabetes mellitus (T2DM), and cardiovascular disorders (Kassi et al., 2011). Obese adolescents, especially those with central adiposity, are more likely to develop glucose intolerance, insulin resistance, dyslipidemia as well as high blood pressure, conditions that comprise the metabolic

\footnotetext{
* Corresponding Author

Moushira Erfan Zaki, Medical Reseaech Dovision,Biological anthropology, Nactional Reseach Centre, Cairo, Egypt.

Email:moushiraz@yahoo.com
}

syndrome. Adipose tissue is an important endocrine organ; it secretes several hormones and cytokines that are involved in the metabolic syndrome. Adipokines, signaling proteins secreted by adipose tissue, have an important regulatory function throughout the body. In obese children low levels $25-(\mathrm{OH}) \mathrm{D}$ were associated with increased markers of oxidative stress, inflammation and endothelial activation (Codoñer-Franch et al., 2012). Lipocalin-2, also known as $24 \mathrm{p} 3$ and neutrophil gelatinase-associated lipocalin (NGAL) (Yan at al., 2007)[3], is a 25-kDa secretory glycoprotein that was originally identified in mouse kidney cells and human neutrophil granules. This protein has been implicated in diversified functions such as apoptosis and innate immunity. In addition to neutrophils, lipocalin-2 is expressed in several other tissues, including liver, lung, kidney, adipocytes, and macrophages (Liu et al.,1995; Kratchmarova et al., 2002; Meheus et al., 1993). Gelatinase-associated Lipocalin (NGAL), which belong to the lipocalin protein family, are associated with insulin resistance (Quadro et al., 1999; Rasouli and Kern, 2008) and metabolic 
syndrome Graham et al.,2006)[9]. Lipocalin-2 was reported to be associated with obesity and insulin resistance (IR) in both mice and humans (Wang et al., 2007).

Previous studies have focused on its role in immune response to bacterial infections (Flo et al., 2004) and as a biomarker of acute kidney failure (Kuwabara et al., 2009). Furthermore, there was a report that expression of lipocalin-2 is increased in atherosclerotic plaques and myocardial infarction and regulates the inflammatory response during ischemia and reperfusion of the transplanted heart in adults (Hemdahl et al., 2006; Aigner et al., 2007).

Studies in animal models have shown that murine atherosclerosis is accompanied by increased levels of serum LCN2 and that conditions of hypoxia and myocardial infarction (MI) induce Lcn2 mRNA expression. Serum LCN2 levels have also been found to be related to glucose metabolism and blood lipid composition. The relationship between lipocalin-2 and obesity is controversial and it has been scarcely studied in children. Therefore, the aim of the current study was to investigate the relationship between serum lipocalin-2 concentrations and metabolic abnormalities in Egyptian obese children.

\section{SUBJECTS AND METHODS}

The present cross-sectional study was conducted during March 2012 to June 2013. The subjects of this study comprised 160 Egyptian children (80 obese and 80 non obese healthy children matched in age and sex). They aged between 6 and 10 years and were prepubertal according to Tanner staging. Inclusion criteria for patients were the existence of obesity as defined by body mass index (BMI) >2 SDS for age and sex upon Egyptian normative charts (http://www.dempuegypt.blogspot.com).

Written informed consent was obtained from all the patients' parents and all investigations followed the Helsinki. This study protocol was approved by the ethical committee board of the National Research Centre of Egypt (No.10/223).

Exclusion criteria for obese children were the presence of endocrinopathy, obesity-associated syndrome, and the presence of any infectious or inflammatory diseases in the past 10 days, or taking medication that affected weight, lipid metabolism, or arterial pressure.

\section{Controls were healthy, lean children prepubertal and matched by age with the obese patients}

\section{Anthropometric measures}

Standing height without shoes was measured twice with a standard scale to the nearest $0.1 \mathrm{~cm}$. Height was measured with the patients standing with their backs leaning against the stadiometer of the same scale. Body weight was measured with the lightest clothing to the nearest $0.1 \mathrm{~kg}$ and body fat percentage (BF \%) was measured by Tanita Body Composition Analyzer (SC-330). BMI was calculated as weight in kilograms divided by height in meters squared $(\mathrm{kg} / \mathrm{m} 2)$. Waist circumference (WC) \& hip circumference
(HC) were measured in $\mathrm{cm}$ using a plastic, non-stretchable tailor's tape. WC was measured with light clothing at a level midway between the lower rib margin and the iliac crest standing and breathing normally. $\mathrm{HC}$ was measured at the level at the widest circumference over the buttocks (at the greater trochanter). The waist hip ratio (WHR) was calculated as WC divided by HC. Mid upper arm circumference (MUAC) measured at a point mid-way between the tip of the shoulder and the tip of the elbow.

The anthropometric measurements and instruments followed the International Biological Programme (IBP) included weight, height, waist and hip circumferences. Measurements were taken three times and the mean values used in the analysis. All circumferences were measured to the nearest $0.1 \mathrm{~cm}$. BMI was calculated as weight in kilograms divided by the square of height in meters.

Blood pressure was measured 3 times with a standard mercury sphygmomanometer and appropriately sized adult cuffs on the right arm of each subject after a 10-minute rest in a sitting position, and the mean values were used for analysis. Skinfold thickness was measured using Holtain Skinfold Caliper is calibrated to $0.2 \mathrm{~mm}$, but the measurement can be conveniently estimated to the last completed $0.1 \mathrm{~mm}$ by the same investigator at the following sites: triceps-half-way between the acromion and the olecranon; and subscapular- $1 \mathrm{~cm}$ below the inferior angle of the scapula dial of the caliper illustrated .

All obese subjects and non obese controls underwent standard physical examination, blood samples were obtained following overnight fasting. Serum total cholesterol, HDLcholesterol and LDL, triglycerides, insulin and glucose were analyzed by routine biochemical procedures. Insulin resistance was assessed at baseline by using the homeostasis model assessment (HOMA). The HOMA-IR was derived as estimates of insulin sensitivity. HOMA-IR was calculated using the formula fasting insulin $(\mathrm{U} / \mathrm{mL}) \mathrm{X}$ fasting glucose $(\mathrm{mmol} / \mathrm{L}) / 22.5$ (Keskin et al., 2005).

\section{Serum lipocalin-2 levels were determined using a Human Lipocalin-2 Quantikine ELISA kit}

\section{Statistical analysis}

Data were expressed as means \pm S.D. We used the Kolmogorov-Smirnov test to evaluate variables for normality. Differences between groups were tested using the unpaired Student's t-test or the Mann-Whitney U test. Wilcoxon rank-sum test (for skewed variables) were used to assess the significance of differences found between the obese cases and controls.

Multiple regression analysis was performed with lipocalin-2 concentrations as a dependent variable and the rest of the variables as independent variables. The stepwise method was used for significant variable selection. Values of $\quad P<0.05$ were considered significant. Data were analyzed using SPSS for Windows (version 16.0; SPSS Inc., Chicago, IL, USA). 
Table 1: Clinical and laboratory characteristics of study subjects.

\begin{tabular}{|c|c|c|c|c|c|c|}
\hline & Boys & & & Girls & & \\
\hline & $\begin{array}{l}\text { Obese } \\
(n=40)\end{array}$ & $\begin{array}{l}\text { Non-obese } \\
(n=40)\end{array}$ & $\mathbf{P}$ & $\begin{array}{l}\text { Obese } \\
(n=40)\end{array}$ & $\begin{array}{l}\text { Non-obese } \\
(n=40)\end{array}$ & $\mathbf{P}$ \\
\hline Age (years) & $10.5 \pm 2.5$ & $16.5 \pm 2.8$ & 0.71 & $11.4 \pm 3.3$ & $16.9 \pm 3.4$ & 0.71 \\
\hline \multicolumn{7}{|l|}{ Anthropometric and clinical variables } \\
\hline BMI $\left(\mathrm{kg} / \mathrm{m}^{2}\right)$ & $22.4 \pm 5.5$ & $13.6 \pm 3.2$ & .001 & $23.4 \pm 4.6$ & $14.6 \pm 4.2$ & .001 \\
\hline $\mathrm{WC}(\mathrm{cm})$ & $96.8 \pm 10.7$ & $72.8 \pm 10.6$ & .001 & $85.8 \pm 12.1$ & $69.8 \pm 10.4$ & .001 \\
\hline WHR & $.97 \pm .9$ & $.79 \pm .1$ & .001 & $.87 \pm .8$ & $.73 \pm .8$ & .001 \\
\hline Mid upper arm circumference $(\mathrm{cm})$ & $31.7 \pm 5.4$ & $20.1 \pm 8.4$ & .001 & $29.7 \pm 6.4$ & $22.6 \pm 5.4$ & .001 \\
\hline Triceps SF (mm) & $30.1 \pm 4.2$ & $17.6 \pm 8.25$ & .001 & $28.1 \pm 5.2$ & $16.3 \pm 5.2$ & .001 \\
\hline Subscapular SF (mm) & $29.4 \pm 5.1$ & $16.1 \pm 6.4$ & .001 & $24.4 \pm 5.9$ & $15.1 \pm 7.4$ & .001 \\
\hline Fat mass $(\mathrm{kg})$ & $28.3 \pm 10.2$ & $14.2 \pm 6.6$ & .001 & $32.3 \pm 14.2$ & $17.2 \pm 6.6$ & .001 \\
\hline Body fat $\%$ & $23.0 \pm 7.7$ & $13.4 \pm 9.0$ & .001 & $25.0 \pm 7.7$ & $14.4 \pm 7.2$ & .001 \\
\hline Systolic BP (mm Hg) & $129.4 \pm 13.7$ & $96.8 \pm 10.4$ & .001 & $125.4 \pm 10.7$ & $89.9 \pm 9.4$ & .001 \\
\hline Diastolic BP (mm Hg) & $74.3 \pm 9.5$ & $66.6 \pm 10.5$ & .001 & $72.3 \pm 9.5$ & $65.4 \pm 10.5$ & .001 \\
\hline \multicolumn{7}{|l|}{ Metabolic variables } \\
\hline Insulin $(\mu \mathrm{U} / \mathrm{mL})$ & $10.5 \pm 4.8$ & $3.51 \pm 4.9$ & .001 & $11.6 \pm 5.9$ & $3.9 \pm 3.9$ & .001 \\
\hline Glucose (mg/dL) & $92.5 \pm 13.5$ & $91.7 \pm 11.3$ & .88 & $91.6 \pm 12.3$ & $90.1 \pm 8.3$ & .61 \\
\hline HOMA-IR & $5.3 \pm 1.5$ & $2.2 \pm 1.4$ & .001 & $6.3 \pm 1.9$ & $1.9 \pm 1.3$ & .001 \\
\hline Cholesterol (mg/dL) & $159.8 \pm 22.4$ & $149.1 \pm 25.2$ & .05 & $155.8 \pm 23.4$ & $145.9 \pm 21.2$ & .04 \\
\hline Triglycerides (mg/dL) & $110.3 \pm 34.0$ & $76.8 \pm 20.1$ & .001 & $108.3 \pm 25.0$ & $76.8 \pm 19.4$ & .001 \\
\hline $\mathrm{HDL}-\mathrm{C}(\mathrm{mg} / \mathrm{dL})$ & $40.9 \pm 12.6$ & $56.7 \pm 10.2$ & .004 & $39.8 \pm 12.6$ & $56.7 \pm 10.2$ & .004 \\
\hline $\mathrm{LDL}-\mathrm{C}(\mathrm{mg} / \mathrm{dL})$ & $132.4 \pm 26.2$ & $100.7 \pm 30.1$ & .02 & $136.4 \pm 30.8$ & $101.7 \pm 28.7$ & 04. \\
\hline \multicolumn{7}{|l|}{ Diagnostic biomarkers } \\
\hline Lipocalin 2 (ng/ml) & $39.7 \pm 16.2$ & $23 \pm 10.66$ & .001 & $38.9 \pm 15.88$ & $24.8 \pm 13.77$ & .001 \\
\hline Leptin $(\mathrm{ng} / \mathrm{mL})$ & $13.56 \pm 7.55$ & $4.8 \pm 2.2$ & .001 & $15.56 \pm 8.55$ & $5.8 \pm 2.2$ & .001 \\
\hline
\end{tabular}

Body mass index; WC: waist circumference; HC: hip circumference; WHR: waist to hip ratio; HDL-C: high-density lipoprotein cholesterol; LDL: low-density lipoprotein cholesterol.

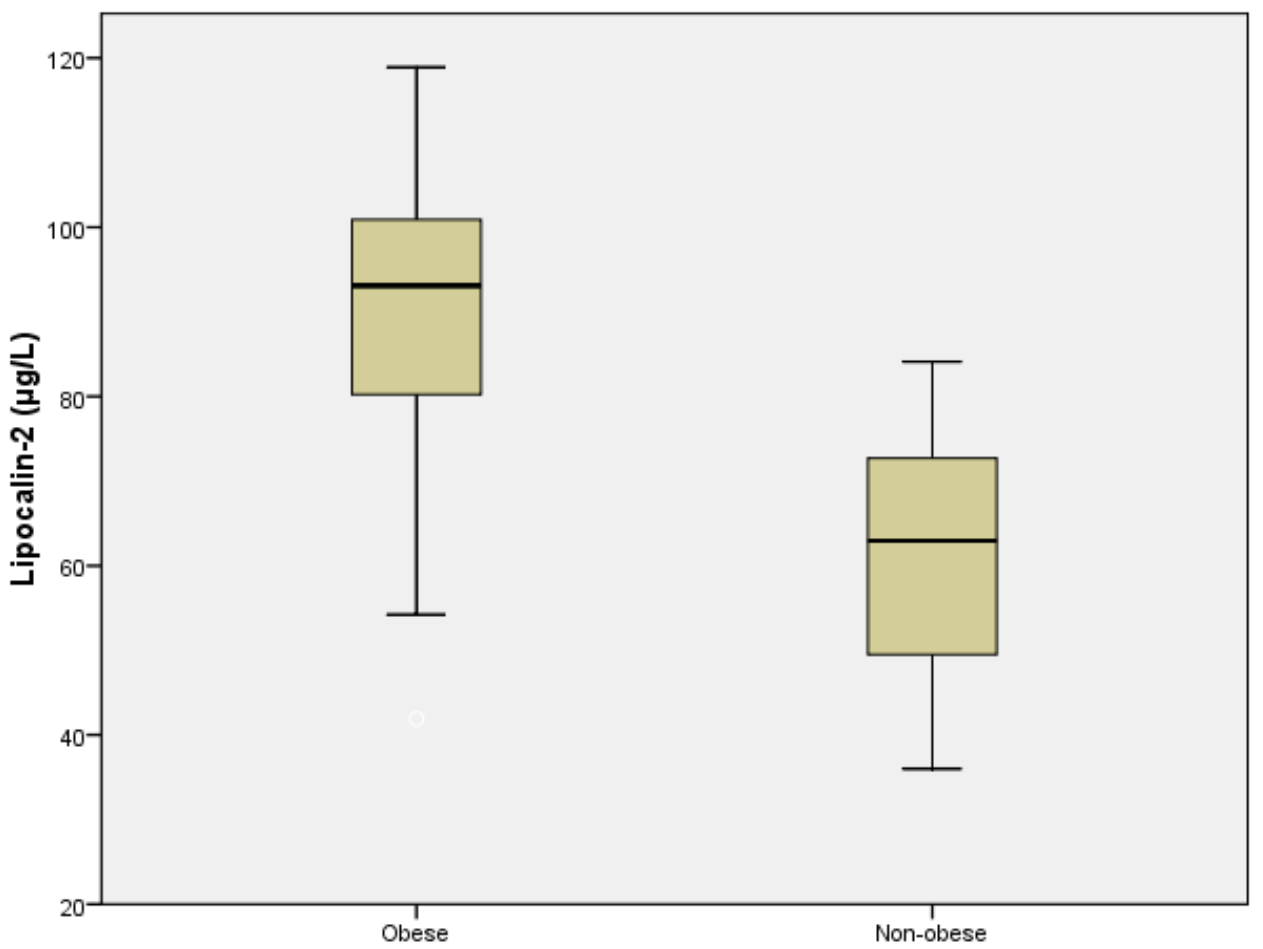

Fig. 1: Box plot of Lipocalin-2 ( $\mu \mathrm{g} / \mathrm{L})$ by BMI category. The box plot displays the 25 th, median and 75 th percentiles, and the minimum and maximum levels as horizontal lines outside the box.

\section{Results}

Clinical and biochemical characteristics of obese and non obese boys and girls are presented in Table 1. Anthropometric parameters, including BMI, waist circumference, WHR, MUAC, skinfolds thickness, fat mass, $\mathrm{BF} \%$, systolic and diastolic $\mathrm{BP}$, insulin, glucose, HOMA-IR, total cholesterol, LDL-C, triglycerides, lipocalin-2 concentration were significantly higher and HDL-C was significantly lower in obese group than non obese controls in both genders. Figure 1 shows pox-plot of lipocalin-2 concentrations according to BMI.

Table 2 shows results of partial correlation analysis between adjusted lipocalin-2 for gender, age and BMI. Results shows that adjusted lipocalin-2 concentrations were positively correlated with weight, waist circumference, body fat \%, systolic 
and diastolic BP, insulin, glucose, and HOMA-IR triglycerides, LDL-C and total cholesterol, whereas they had a negative correlation with HDL-C.

The stepwise multiple regression analysis showed that lipocalin-2 levels were independently associated with LDL-C, triglycerides, total cholesterol and HOMA-IR (Table 3).

Table2: Partial correlation analyses of Lipocalin-2, adjusted for age, gender and body mass index.

\begin{tabular}{lcc}
\hline & \multicolumn{3}{c}{ Lipocalin-2 } \\
\hline Weight $(\mathrm{kg})$ & $\mathbf{r}$ & $\mathbf{P}$ \\
Age & .45 & .025 \\
WC & .039 & .511 \\
WHR & .496 & .001 \\
MUAC & .055 & .25 \\
Triceps SF (mm) & .08 & .892 \\
Subscapular SF (mm) & .0526 & .52 \\
Body Fat \% & .069 & .32 \\
Systolic BP (mm Hg) & .664 & .024 \\
Diastolic BP (mm Hg) & .451 & .001 \\
Insulin $(\mu \mathrm{m} / \mathrm{mL})$ & .675 & .001 \\
Glucose $(\mathrm{mg} / \mathrm{dL})$ & .622 & .001 \\
HOMA-IR & .593 & .001 \\
Cholesterol (mg/dL) & .664 & .001 \\
Triglycerides $(\mathrm{mg} / \mathrm{dL})$ & .463 & .022 \\
HDL-C (mg/dL) & .551 & .041 \\
LDL-C (mg/dL) & -.622 & .001 \\
\hline
\end{tabular}

Body mass index; WC: waist circumference; HC: hip circumference; WHR: waist to hip ratio; MUAC: mid upper arm circumference; HDL-C: high-density lipoprotein cholesterol; LDL: low-density lipoprotein cholesterol; HOMA-IR, homeostasis model assessment insulin resistance.

Table 3: Multiple regression analysis with lipocalin-2 concentrations as dependent variable.

\begin{tabular}{|c|c|c|c|c|}
\hline \multicolumn{5}{|c|}{ lipocalin-2 } \\
\hline & \multicolumn{2}{|c|}{ Unstandardized Coefficients } & \multicolumn{2}{|c|}{ Standardized Coefficients } \\
\hline & $\beta$ & S.E & Standard $\beta$ & p \\
\hline $\mathrm{LDL}-\mathrm{C}$ & 256. & .230 & 293. & .004 \\
\hline Cholesterol & .34 & .211 & .355 & .002 \\
\hline Triglycerides & .24 & .611 & .859 & .002 \\
\hline HOMA-IR & 754. & 115. & 593. & .003 \\
\hline
\end{tabular}

\section{DISCUSSION}

In the current study, we investigated lipocalin-2 as diagnostic biomarkers for metabolic abnormalities which related to obesity in pre-pubertal children.

Results show that obese children had significantly higher mean serum concentrations of lipocalin-2 than non obese controls. Furthermore, the study observed that after adjustment of lipocalin2 for gender, age and BMI correlation between lipocalin-2 concentrations and obesity parameters remained significant, whereas with metabolic risk variables such as systolic diastolic blood pressure; fasting serum concentrations of insulin, whereas triglycerides, glucose and HOMA-IR and HDL-c

To further understand the relationship between lipocalin2 with the metabolic syndrome risk variables, we carried out the multiple regression analysis which showed that this biomarker was independently associated metabolic syndrome components.

Previous studies have suggested contradictory roles for lipocalin-2 in glucose metabolism. A linear association between Lipocalin-2 and 25- (OH) D, as well as between Retinol-Binding-
Protein-4 and 25-(OH) D, is evident in the results of previous obese cases. Studies of Gavi et al., (2007) show a positive correlation between RBP4, adiposity and an increased inflammatory state. There are other studies, though, that do not support the above (Promintzer et al., 2007; Broch et al., 2007).

Other cross-sectional human studies reported contradictory roles for lipocalin-2 in glucose metabolism in old subjects. In a study of 33- to 72-year-old men and women, a positive correlation between lipocalin-2 and adiposity, triglycerides, glucose, and HOMA-IR was found. These correlations were significant even after adjusting age, sex, and BMI. However, a study of 58-year-old healthy Swedish men found no correlation between lipocalin-2 and insulin sensitivity, waist circumference, or BMI, although it showed an inverse correlation with HDL- and LDL-cholesterol (Wallenius et al., 2011) .

One study using visceral fat from obese subjects did confirm lipocalin-2 mRNA expression in this tissue, but subcutaneous fat tissue was not studied (Catalan et al., 2009). Interventional studies looking at the effect of weight loss, which is known to improve insulin sensitivity, have mostly found no change in lipocalin-2 levels with weight loss (Corripio et al., 2010; Choi et al., 2009).

In a 2-year longitudinal study that evaluates the association between plasma concentrations of lipocalin-2 and metabolic syndrome parameters in prepubertal children, before and after a diet and lifestyle intervention program reported that lipocalin-2 in obese pre-pubertal children showed a $44.7 \%$ higher concentration than that in lean children, after controlling age and sex. However, it did not significantly decrease after weight loss in obese patients after 2 years of follow-up (Wang et al., 2007).

Lipocalin-2 seems to act as an acute phase protein, which can be induced by different inflammatory processes (Lin et al., 2001). In obese states, these proinflammatory factors are produced predominantly from enlarged adipocytes and activated macrophages in adipose tissue and liver. Many of these inflammatory factors, such as interleukin (IL) 6, tumor necrosis factor alpha, and resistin, can directly induce glucose intolerance and insulin resistance by antagonizing insulin's metabolic actions at peripheral tissues, especially in liver and skeletal muscle (Trayhurnet al., 2004; Fantuzzi et al., 2005). On the other hand, several other adipokines produced from adipocytes, including adiponectin and visfatin, have demonstrated insulin-sensitizing activity and exertion of beneficial effects on glucose and lipid homeostasis (Kadowaki 2005; Kadowaki and Yamauchi, 2005; Fukuhara et al., 2005). Expression of lipocalin-2 in both adipose tissue and liver can be induced by lipopolysaccharides, suggesting lipocalin-2 to be an acute phase protein (Lin et al., 2001). Likewise, in our study, lipocalin-2 concentrations did not significantly decrease after weight loss in obese patients after 2 years of follow-up. Lipocalin-2 appears to act as an acute phase protein, which can be induced by different inflammatory processes (Wang et al., 2007). In obesity, it has been shown that lipocalin-2 is an independent predictor of inflammation and it correlates with PCR after adjusting for age, sex, and adiposity (Zografos et al., 
2009). The possible association of serum LCN2 levels and coronary artery disease (CAD) has been addressed by clinical trials, and studies based on Caucasian and Korean populations have suggested positive associations between expression of this multifunctional protein and development of this life threatening disease with systemic implications. However, no study to date has assessed the relationship between serum LCN2 levels and CAD in a Chinese population (Choi et al., 2008; Matsubara et al., 2002)

Insulin and leptin are secreted in direct proportion, and adiponectin in negative proportion, to the size of the adipose mass. These three hormones are key molecules in the regulation of lipid metabolism. During states of positive energy balance, as it occurs in obesity, the adipose mass expands and more leptin and insulin but less adiponectin are secreted (Fried et al., 2000). Leptin and insulin reach the brain and, as a consequence, anabolic pathways are inhibited and catabolic pathways are activated. These two hormones, as well as adiponectin, also interact with peripheral tissue receptors modulating energy metabolism. Leptin is produced by adipose tissue and considered to be one of the main peripheral signals that regulate food intake, energy expenditure and body weight, by reporting nutritional information to key regulatory centers in the hypothalamus. Leptin exerts complex effects on the storage and metabolism of fats and carbohydrates. These are mediated both directly, through actions on specific tissues, and indirectly, through CNS endocrine and neural mechanisms.

Leptin stimulates fatty acid oxidation and glucose uptake and prevents lipid accumulation in non-adipose tissues, which can lead to lipotoxicity and functional impairments. The increase in the expression of uncoupling proteins (UCP) in adipose tissue and muscle, which occurs during leptin treatment may also account for leptin's ability to prevent the decrease in energy expenditure that takes place during the reduction of food intake.

There is a controversy about the direct interaction between leptin and insulin. Apparently, hyperinsulinemia promotes fat deposition, which subsequently increases leptin expression (Girard et al., 1997). Leptin has also been suggested to be related to insulin resistance. Moreover, in healthy children and adults, obese individuals or those who have insulin-dependent or non-insulin-dependent diabetes, there is a positive correlation between leptin and insulin concentrations during fasting, independent of body fat content. These results led to the suggestion that leptin might be the signal from adipocytes to islets to hypersecrete insulin when fat content is increased and insulin sensitivity is lowered (Larsson et al., 1996; Valle et al., 2003). However, a great deal of additional information is needed to clarify the relationship between leptin and insulin sensitivity, in both physiological and pathological conditions.

In human obese subjects, like other insulin resistanceinducing adipokines and cytokines, circulating lipocalin-2 levels are markedly elevated (Zhang et al., 2008).

In summary, our study provides clinical evidence demonstrating that serum concentrations of lipocalin-2 are closely associated with obesity and its related metabolic complications. The present study suggests that the measurement of this biomarker may be useful for evaluating metabolic syndrome in Egyptian obese children.

\section{REFERENCES}

Aigner F, Maier HT, Schwelberger HG, Wallnofer EA, Amberger A, Obrist P, Berger T, Mak TW, Maglione M, Margreiter R, Schneeberger S \& Troppmair J. Lipocalin-2 regulates the inflammatory response during ischemia and reperfusion of the transplanted heart. American Journal of Transplantation 2007; 7779-788.

Broch M, Vendrell J, Ricart W, Richart C, Fernández-Real JM, Circulating retinol-binding protein-4, insulin sensitivity, insulin secretion, and insulin disposition index in obese and non obese subjects. Diabetes Care 2007; 30: 1802-1806.

Catalan V, Gomez-Ambrosi J, Rodriguez A, Ramirez B, Silva C, Rotellar F, Gil MJ, Cienfuegos JA, Salvador J \& Fruhbeck G. Increased adipose tissue expression of lipocalin-2 in obesity is related to inflammation and matrix metalloproteinase-2 andmetalloproteinase-9 activities in humans. Journal of Molecular Medicine 2009; 87: 803-813.

Choi KM, Kim TN, Yoo HJ, Lee KW, Cho GJ, Hwang TG, Baik SH, Choi DS \& Kim SM. Effect of exercise training on A-FABP, lipocalin-2 and RBP4 levels in obese women. Clinical Endocrinology 2009; 70: 569-574.

Choi KM, Lee JS, Kim EJ, Baik SH, Seo HS, Choi DS, Oh DJ, Park CG: Implication of lipocalin-2 and visfatin levels in patients with coronary heart disease. Eur J Endocrinol 2008, 158:203-207.

Codoñer-Franch P, Tavárez-Alonso S, Simó-Jordá R, LaportaMartín P, Carratalá-Calvo A, Alonso-Iglesias E, Vitamin D status is linked to biomarkers of oxidativestress, inflammation, and endothelial activation in obese children. J Pediatr 2012; 161: 848-854.

Corripio R, Gonzalez-Clemente JM, Perez-Sanchez J, Naf S, Gallart L, Nosas R, Vendrell J \& Caixas A. Weight loss in prepubertal obese children is associated with a decrease in adipocyte fatty-acidbinding protein without changes in lipocalin-2: a 2-year longitudinal study. European Journal of Endocrinology 2010; 163: 887-893.

Fantuzzi G. Adipose tissue, adipokines, and inflammation. J Allergy Clin Immunol 2005; 115: 911-9.

Flo T, Smith K, Sato S, Rodriguez D, Holmes M, Strong R, Akira $\mathrm{S} \&$ Aderem A. Lipocalin 2 mediates an innate immune response to bacterial infection by sequestrating iron. Nature 2004; 432: 917-921.

Fried SK, Ricci MR, Russell CD, Laferre're B. Regulation of leptin production in humans. J Nutr 2000; 130: 3127-3131.

Fukuhara A, Matsuda M, Nishizawa M, Segawa K, Tanaka M, Kishimoto K, et al. Visfatin: a protein secreted by visceral fat that mimics the effects of insulin. Science 2005; 307: 426-30.

Gavi S, Stuart LM, Kelly P, et al., Retinol-binding protein 4 is associated with insulin resistance and body fat distribution in non-obese subjects without type 2 diabetes. J Clin Endocrinol Metab 2007; 92: 18861890.

Girard J. Is leptin the link between obesity and insulin resistance? Diabetes Metab 1997; 23 (Suppl 3): 16-24.

Graham TE, Yang Q, Blucher M, et al. Retinol-Binding Protein 4 and Insulin Resistance. N Engl J Med 2006; 354: 2552-2563.

Hemdahl AL, Gabrielsen A, Zhu C, Eriksson P, Hedin U, Kastrup J, Thoren P \& Hanssor GK. Expression of neutrophil gelatin aseassociated lipocalin in atherosclerosis and myocardial infarction. Arteriosclerosis, Thrombosis, and Vascular Biology 2006; 26: 136-142.

Kadowaki T, Yamauchi T. Adiponectin and adiponectin receptors. Endocr Rev 2005; 26: 439-51.

Kassi E, Pervanidou P, Kaltsas G, Chrousos G, Metabolic Syndrome Definitions and Controversies. BMC Medicine 2011; 9:48.

Keskin M, Kurtoglu S, Kendirci M, et al: Homeostasis model assessment is more reliable than the fasting glucose/insulin ratio and quantitative insulin sensitivity check index for assessing insulin resistance among obese children and adolescents. Pediatr; 2005, 115:e500-e503.

Kobayashi K. Adipokines: therapeutic targets for metabolic syndrome. Curr Drug Targets 2005; 6: 525-9.

Kratchmarova I, Kalume DE, Blagoev B, Scherer PE, Podtelejnikov AV, Molina $\mathrm{H}$, et al. A proteomic approach for 
identification of secreted proteins during the differentiation of 3T3-L1 preadipocytes to adipocytes. Mol Cell Proteomics 2002; 1: 213-22.

Kuwabara T, Mori K, Mukoyama M, Kasahara M, Yokoi H, Saito Y, Yoshioka T, Ogawa Y, Imamaki H, Kusakabe T, Ebihara K, Omata M, Satoh N, Sugawara A, Barasch J \& Nakao K. Urinary neutrophil gelatinase-associated lipocalin levels reflect damage to glomeruli, proximal tubules, and distal nephrons. Kidney International 2009; 75: 285-294.

Larsson H, Elmstahl S, Ahren B. Plasma leptin levels correlate to islet function independently of body fat in postmenopausal women. Diabetes 1996; 45: 1580-1584.

Lin Y, Rajala M, Berger J, Moller D, Barzilai N \& Scherer P. Hyperglycemia-induced production of acute phase reactantsin adipose tissue. Journal of Biological Chemistry 2001; 276: 42077-42083.

Liu Q, Nilsen-Hamilton M. Identification of a new acute phase protein. J Biol Chem 1995; 270: 22565-70.

Matsubara M, Maruoka S, Katayose S. Inverse relationship between plasma adiponectin and leptin concentrations innormal-weight and obese women. Eur J Endocrinol 2002; 147: 173-180.

Meheus LA, Fransen LM, Raymackers JG, Blockx HA, Van Beeumen JJ, Van Bun SM, et al. Identification by microsequencing of lipopolysaccharide-induced proteins secreted by mouse macrophages. J Immunol 1993; 151: 1535-47.

Metheniti D, Sakka S, Dracopoulou M, Margeli A, Papassotiriou I, Kanaka-Gantenbein C, Chrousos GP, Pervanidou P. Decreased circulating 25-(OH) Vitamin D concentrations in obese female children and adolescents: positive associations with Retinol Binding Protein-4 and Neutrophil Gelatinase-associated Lipocalin Hormones 2013; 12: 397-404.

Promintzer M, Krebs M, Todoric J, et al. Insulin resistance is unrelated to circulating retinol binding protein and protein $\mathrm{C}$ inhibitor. $\mathrm{J}$ Clin Endocrinol Metab 2007; 92: 4306-4312.

Quadro L, Blaner WS, Salchow DJ, et al. Impaired retinal function and vitamin A availability in mice lacking retinol-binding protein. EMBO J 1999; 18: 4633-4644.

Rasouli N, Kern PA, Adipocytokines and the metabolic complications of obesity. J Clin Endocrinol Metab 2008; 93: 64-73.
Trayhurn P, Wood IS. Adipokines: inflammation and the pleiotropic role of white adipose tissue. Br J Nutr 2004; 92: 347-55.

Valle M, Gascón F, Martos R, Bermudo F, Ceballos P, Suanes A. Relationship between high plasma leptin concentrations and metabolic syndrome in obese pre-pubertal children. Int J Obes Relat Metab Disord 2003; 27 : 13-18.

Wallenius V, Elias E, Bergstrom GM, Zetterberg H \& Behre CJ. The lipocalins retinol-binding protein-4, lipocalin-2 and lipocalin-type prostaglandin D2-synthase correlate with markers of inflammatory activity, alcohol intake and blood lipids, but not with insulin sensitivity in metabolically healthy 58-year-old Swedish men. Experimental and Clinical Endocrinology \& Diabetes 2011; 11975-80.

Wang Y, Lam KS, Kraegen EW, Sweeney G, Zhang J, Tso AW, Chow WS, Wat NM, Xu JY, Hoo RL \& Xu A. Lipocalin-2 is an inflammatory marker closely associated with obesity, insulin resistance, and hyperglycemia in humans. Clinical Chemistry 2007; 53: $34-41$.

Yan QW, Yang Q, Mody N, et al, The Adipokine Lipocalin 2 Is Regulated by Obesity and Promotes Insulin Resistance. Diabetes 2007; 56 : 2533-2540.

Zhang J, Wu Y, Zhang Y, Leroith D, Bernlohr DA, Chen X. The role of lipocalin 2 in the regulation of inflammation in adipocytes and macrophages Mol Endocrinol 2008;22:1416-1426.

Zografos T, Haliassos A, Korovesis S, Giazitzoglou E, Voridis E, Katritsis D: Association of neutrophil gelatinase-associated lipocalin with theseverity of coronary artery disease. Am J Cardiol 2009, 104:917920.

\section{How to cite this article:}

Moushira Erfan Zaki, Hala El-Bassyouni, Eman Youness, Nadi Mohamed. Lipocalin-2 is an inflammatory biomarker associated with metabolic abnormalities in Egyptian obese children. J App Pharm Sci, 2015; 5 (05): 007-012. 